\title{
Predictors of Quality of Life Among Breast Cancer Patients
}

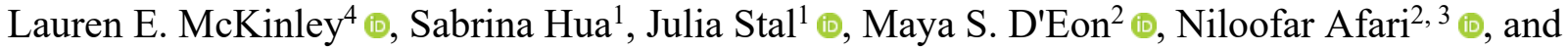 \\ Terry A. Cronan ${ }^{1}$ it \\ ${ }^{1}$ San Diego State University, San Diego, California, United States \\ ${ }^{2}$ Department of Psychiatry, University of California San Diego, La Jolla, United States \\ ${ }^{3}$ VA San Diego Healthcare System, San Diego, California, United States \\ ${ }^{4}$ University of California, Merced, United States
}

\begin{abstract}
Background and Purpose: Over 3.1 million women living in the U.S. have been diagnosed with breast cancer. The purpose of the present study was to determine whether stage of cancer, psychological distress, cognitive fusion (fusion of one's thoughts and emotions), mindfulness, and resilience were related to quality of life among breast cancer patients. Method: Participants were 24 women, aged 41-71, diagnosed with stage 0-3 breast cancer, recruited to participate in a pilot intervention study. All data for this study were collected at the baseline assessment. Results: A multiple linear regression analysis explained $79.1 \%$ of the total variance in quality of life, $F(5,14)=15.400, p<.001$, Adjusted $\mathrm{R}^{2}=.791$. Higher resilience significantly predicted higher quality of life $(\mathrm{b}=2.392, p=.002)$, whereas higher stage of cancer $(\mathrm{b}=$ $8.068, p=.030)$ and higher levels of psychological distress $(\mathrm{b}=-1.737, p<.001)$ significantly predicted lower quality of life. Contrary to the hypotheses, higher levels of mindfulness significantly predicted lower quality of life $(\mathrm{b}=-0.612, p=.022)$. Cognitive fusion did not significantly predict quality of life $(p>.05)$. Conclusion: More research is needed to determine how various factors predict quality of life among breast cancer patients.
\end{abstract}

(C) 2021 and CC-BY 4.0 licensed by the authors.

Keywords: breast cancer, quality of life, resilience, distress, mindfulness, cognitive fusion

\section{Introduction}

Breast cancer is the most commonly diagnosed cancer among American women (American Cancer Society, 2017), with over 3.1 million women living in the United States affected (U.S. Breast Cancer Statistics, 2018). Being diagnosed with breast cancer may profoundly impact an individual's quality of life (Mols et al., 2005; Carver et al., 2006). Reduced quality of life has been related to negative treatment outcomes (Stanton et al., 2000; Kershaw et al., 2004). Negative outcomes include reduced self-efficacy and coping capacity (Stanton et al., 2000; Kershaw, Northouse, Kritpracha, Schafenacker,
\& Mood, 2004), along with poorer treatment responses and survival durations (Costanzo et al., 2007; Ganz et al., 2002). These disruptions have been shown to persist long after the completion of treatment (Hoffman, Zevon, D'Arrigo, \& Cecchini, 2004) and may shape breast cancer patients' perceptions of how cancer impacted their lives (Zebrack, Yi, Petersen, \& Ganz, 2008). Researchers have found that both physical and psychological factors contribute to changes in breast cancer patients' overall quality of life (Mols, Vingerhoets, Coebergh \& van de PollFranse, 2005; Carver, Smith, Petronis, \& Antoni, 2006). 
The stage of breast cancer with which an individual is diagnosed is broadly determined by tumor size, number of lymph nodes affected, and whether the cancer has invaded other organs in the body and to what extent (National Breast Cancer Foundation, Inc., 2016). More advanced stages of breast cancer have predicted lower quality of life among affected individuals (Montazeri, 2008). One notable reason is that women diagnosed with more advanced stages of breast cancer often require more invasive treatments like chemotherapy and/or chemoimmunotherapy (Lemieux, Maunsell, \& Provencher, 2008; Kröger, Menzel, Gschwend, \& Bergmann, 1999). Researchers have also found that breast cancer patients who undergo total mastectomies, as opposed to partial mastectomies, often experience lower quality of life, and this is especially true for women under the age of 50 (Ganz, Greendale, Petersen, Kahn, \& Bower, 2003). Advanced stages of breast cancer are associated with increased levels of distress and anxiety, resulting in lower quality of life (Hoffman, Zevon, D’Arrigo, \& Cecchini, 2004). Therefore, stage of cancer is a diseaserelated factor that may predict patients' overall quality of life and have both physical and psychological effects on the individual.

Researchers have identified several psychological predictors of quality of life among women diagnosed with breast cancer (Mols et al., 2005; Carver et al., 2006). One such predictor is psychological distress (Härtl, Engel, Herschbach, Reinecker, Sommer \& Friese, 2010; Hegel et al., 2006). High levels of psychological distress may interfere with coping ability and negatively impact treatment outcomes, ultimately impacting quality of life (Hoffman et al., 2004). For some women, high levels of distress may persist long after their treatment has been completed (Costanzo et al., 2007). Thus, psychological distress has been shown to affect individuals' quality of life and, for some, these effects may be long-lasting.
Cognitive fusion has been defined as the degree to which individuals are attached to their thoughts, or the failure to disconnect one's thoughts from one's experiences (Solé et al., 2016). Fusion with cancer-related cognitions and associated avoidant coping behaviors have been shown to predict lower quality of life among cancer patients (Gillanders et al., 2015). Some have postulated that cognitive fusion leads to psychological inflexibility, which may exacerbate feelings of psychological distress, and thereby negatively impact quality of life (Hayes, Luoma, Bond, Masuda, \& Lillis, 2006; Fergus \& Bardeen, 2016; Gillanders, Sinclair, MacLean, \& Jardine, 2015). Thus, cognitive fusion has been shown to impact breast cancer patients' quality of life.

Increased mindfulness has been associated with improved mental health (Grossman, Niemann, Schmidt, \& Walach, 2004). Researchers have found that breast cancer patients who participated in mindfulness-based meditation for stress reduction experienced improved quality of life (Carlson, Speca, Patel \& Goodey, 2003; Lengacher et al., 2009; Witek-Janusek et al., 2008; Hoffman et al., 2012; Henderson et al., 2012). However, researchers have defined mindfulness differently, making it difficult to compare across studies (Coffey, Hartman, \& Fredrickson, 2010). Moreover, in the mindfulness-based meditation studies, participants were taught how to engage in mindfulness techniques. Thus, additional research is needed to determine the effects of mindfulness on individuals' quality of life at baseline without prior education or coaching.

Resilience has been defined as an individual's ability to protect their mental health when faced with adversity (Ristevska-Dimitrovska, Filov, Rajchanovska, Stefanovski, \& Dejanova, 2015). Resilience has been shown to predict higher quality of life among breast cancer patients (Ha, Jung, \& Choi, 2014). Researchers found that higher levels of resilience were related to higher 
quality of life among both adolescent and adult cancer patients ( $\mathrm{Wu}$ et al., 2015). Additionally, higher levels of resilience have been related to better psychological outcomes in both women with breast cancer and women in a control group, but this association was stronger among the cancer patients (Markovitz, Schrooten, Arntz \& Peters, 2015).

As advancements in medicine and technology continue to improve breast cancer patients' physical outcomes, it is crucial that researchers examine factors to improve psychological outcomes. To do this, researchers must gain a thorough understanding of how various combinations of physical and psychological factors predict quality of life. The consideration of these contributing factors will become an important aspect of developing effective therapeutic techniques to ensure optimal patient quality of life. Although stage of cancer, resilience, and distress have been shown to predict quality of life among breast cancer patients, cognitive fusion and mindfulness have yet to be evaluated in this context. The present study was exploratory. The purpose was to determine whether stage of cancer, psychological distress, cognitive fusion, mindfulness, and resilience predicted quality of life among a small number of breast cancer patients, so that the results could be used to inform the development of larger studies. It was hypothesized that higher levels of mindfulness and resilience would predict higher quality of life, and that higher stage of cancer, cognitive fusion, and psychological distress would predict lower quality of life.

\section{Method}

\section{Participants}

The participants were 24 women diagnosed with stage 0-3 breast cancer who were recruited to participate in a pilot intervention study. The data for the present study were collected at the baseline assessment. To be eligible, participants were required to have been diagnosed with breast cancer within the past year, have already completed curative surgery (if applicable to their treatment plan), be at least 21 years old, have had no previous cancer diagnoses (with the exception of non-melanoma skin cancer), and score 13 or higher on the 18-item Brief Symptom Inventory (BSI-18), indicating the presence of psychological distress. The cutoff score of 13 or higher was based on data from normed adult female oncology patients (Zabora, Brintzenhofeszoc, Curbow, Hooker, \& Piantadosi, 2001). Of the 24 women who participated in the study, 23 had received some form of surgical intervention for their breast cancer. Age of participants ranged from 41 to 71 years $\left(M_{\text {age }}=55.38, S D=8.5\right)$. The majority of participants were Caucasian $(91.7 \%)$, had at least a bachelor's degree (50\%), and an annual family income of $\$ 80,000$ or more $(58 \%)$. Additional participant demographic information is reported in Table 1.

Table 1. (cont'd)

Participant Demographic Characteristics

\begin{tabular}{lll}
\hline Item & $\mathrm{N}$ & Valid \% \\
\hline Race & & \\
$\quad$ White & 22 & 91.67 \\
$\quad$ Black & 1 & 4.17 \\
$\quad$ Not Reported & 1 & 4.17 \\
Ethnicity & & \\
$\quad$ Hispanic or Latino & 5 & 20.83 \\
$\quad$ Not Hispanic or Latino & 15 & 62.50 \\
$\quad$ Not Reported & 4 & 16.67 \\
& & \\
Education & & \\
$\quad$ High School Graduate or Less & 1 & 4.17 \\
$\quad$ Some College/Associate's & 11 & 45.83 \\
$\quad$ Bachelor's Degree & 6 & 25.00 \\
$\quad$ Graduate Level Degree & 6 & 25.00
\end{tabular}


Table 1. (cont'd)

Participant Demographic Characteristics

\begin{tabular}{lll}
\hline Item & $\mathrm{N}$ & Valid \% \\
\hline Income & & \\
$\quad \$ 10,001-\$ 30,000$ & 3 & 12.50 \\
$\$ 30,001-\$ 50,000$ & 3 & 12.50 \\
$\$ 50,001-\$ 80,000$ & 4 & 16.67 \\
$\$ 80,001-\$ 110,000$ & 6 & 25.00 \\
$\$ 110,001$ or more & 8 & 33.33 \\
& & \\
Employment Status & & \\
Full-Time & 4 & 16.67 \\
Part-Time & 6 & 25.00 \\
Unemployed & 3 & 12.50 \\
Retired & 4 & 16.67 \\
Disabled & 2 & 8.33 \\
Other & 5 & 20.83 \\
Relationship Status & & \\
Single & 3 & 12.5 \\
In a Relationship & 2 & 8.33 \\
Married & 14 & 58.33 \\
Separated/Divorced & 5 & 20.83 \\
Participant Stage of Cancer & & \\
0 & 3 & 12.50 \\
1 & 12 & 50.00 \\
2 & 6 & 25.00 \\
3 & 3 & 12.50 \\
\hline
\end{tabular}

\section{Design}

A cross-sectional study design was employed. The independent variables were stage of cancer, psychological distress, cognitive fusion, mindfulness, and resilience. The dependent variable was quality of life. These constructs were measured at baseline prior to the participants' exposure to the intervention. Descriptive statistics for each of the measures assessing the independent variables and the dependent variable are provided in Table 2. For each measure, mean, standard deviation, minimum score and maximum score were reported.

Table 2.

Descriptive Statistics of Summed Scores

\begin{tabular}{lrrrr}
\hline Measure (N) & Mean & SD & $\begin{array}{r}\text { Min } \\
\text { Score }\end{array}$ & $\begin{array}{r}\text { Max } \\
\text { Score }\end{array}$ \\
\hline CFQ-13 (22) & 43.00 & 11.92 & 20 & 63 \\
BSI-18 (20) & 26.73 & 12.67 & 9 & 54 \\
FFMQ (22) & 95.59 & 28.46 & 25 & 141 \\
CD-RISC & 28.96 & 6.82 & 7 & 37 \\
$(22)$ & & & & \\
FACT-B (22) & 95.59 & 28.46 & 25 & 141 \\
\hline
\end{tabular}

Measures

Stage of Cancer. A medical history questionnaire was administered to participants to obtain detailed information about their cancer diagnosis and demographic information. The demographic information included age, race, ethnicity, marital status, employment, education, and annual household income. Participants were asked to list their medications and whether they had comorbid health conditions. Participants were also asked to provide the following details about their cancer diagnosis: type of breast cancer, stage of breast cancer, completed treatments, and planned treatments. Types of treatments included surgical procedures, chemotherapy, radiation, medications, and alternative treatments (e.g. acupuncture). In addition, participants were asked to provide a rating of their overall health using a 5-point Likert-type scale ranging from 1 (Very poor) to 5 (Excellent). Stage of cancer was analyzed as a predictor of quality of life.

Psychological Distress. The Brief Symptom Inventory 18 (BSI-18) is an 18-item measure of overall distress, with subscales measuring depression, anxiety, and somatization (Derogatis, 
2000). The BSI has demonstrated high internal consistency $(\alpha=0.89)$ and has been normed with oncology samples. A score of 13 or higher indicates the presence of psychological distress among adult female oncology patients (Zabora et al., 2001). The Cronbach's alpha coefficient from our sample indicated high internal consistency $(\alpha$ $=0.913$ ).

Cognitive Fusion. The Cognitive Fusion Questionnaire (CFQ-13) is a 13-item measure of cognitive fusion, where higher scores indicate increased cognitive fusion. It has demonstrated strong internal consistency $(\alpha=0.88$; Gillanders et al., 2014; McCracken, Skillicorn, Doherty, 2014). The Cronbach's alpha coefficient from our sample indicated high internal consistency $(\alpha=$ 0.885).

Mindfulness. The Five-Facet Mindfulness Questionnaire (FFMQ) was used. The FFMQ was developed from a factor analysis of five independent mindfulness questionnaires to measure the five underlying components of mindfulness, including observing, describing, acting with awareness, non-judging of inner experience, and non-reactivity to inner experience. Internal consistency of the measure ranged from moderate to high $(\alpha=.67$ to .92 ; Baer et al., 2008). Scoring of the FFMQ yielded five subscale scores, one for each component, and an overall total score, which was used in the analysis for the current study. Higher scores indicate greater mindfulness (total scores range from 39 to 195). The Cronbach's alpha coefficient from our sample indicated high internal consistency $(\alpha=0.930)$.

Resilience. The Connor-Davidson Resilience Scale (CD-RISC) is a 10-item measure of resilience, where higher scores indicate greater resilience. The CD-RISC has demonstrated adequate test-retest reliability across an interval of two months $(r=.66)$ and high internal consistency ( $\alpha=0.81$ to 0.94 ; Xie, Peng, Zuo \& $\mathrm{Li}, 2016)$. The Cronbach's alpha coefficient from our sample indicated high internal consistency ( $\alpha$ $=0.914)$.

Outcome Variable (Quality of Life). The Functional Assessment of Cancer Therapy-Breast Version 4 (FACT-B) is a scale consisting of 42 items with five subscales measuring physical, social/familial, emotional, functional, additional concerns regarding breast cancer-specific wellbeing, and arm morbidity. The FACT-B has indicated high internal consistency $(\alpha=.90)$, high test-retest reliability over a period of three to seven days $(\mathrm{r}=.85)$, and adequate construct validity (Brady et al., 1997). The overall total score was used for the current study (total scores range from 0 to 168) and higher scores indicate greater quality of life. The Cronbach's alpha coefficient from our sample indicated high internal consistency $(\alpha=0.882)$.

\section{Procedures}

After receiving the appropriate Institutional Review Board approvals, a list of mailing addresses was obtained from the California Cancer Registry of patients diagnosed with stage 0 to 3 breast cancer, within the past year, in San Diego County. Recruitment letters were mailed to the homes of eligible patients over a two-month period asking whether they were interested in participating in a 3-month long intervention study for breast cancer patients. Each letter described the purpose of the study and instructed interested individuals to call for more information regarding eligibility criteria. A total of 629 letters were mailed to potential participants. Only one letter was sent to each potential participant to maintain confidentiality. Forty-six women called to inquire about the study. Women who called were asked to complete a phone screening process that included a verbal administration of the BSI-18 to determine whether they were experiencing psychological distress and to confirm that they met the other eligibility criteria (e.g., that they could attend eight weekly 90 -minute intervention group meetings). Twenty-nine of the women screened by phone qualified for the study. Five of 
the women who qualified for the study were either not interested in participating after hearing about the study or did not show up for their initial assessment and did not return calls for rescheduling their assessment. Thus, 24 (83\%) women who met the aforementioned criteria and agreed to participate in the study completed the initial assessment.

During the assessment, a trained research assistant described the details of the study to interested individuals prior to obtaining their written consent to participate. Next, the research assistant administered a medical history questionnaire. After the medical history questionnaire, participants completed a series of standardized self-report measures, and upon completion were compensated $\$ 25.00$. The study was approved by the university's Institutional Review Board and from the State of California, and signed consent was obtained from all participants.

\section{Statistical Analyses}

A multiple linear regression analysis was conducted to examine whether stage of cancer, psychological distress, cognitive fusion, mindfulness, and resilience predicted quality of life among breast cancer patients. Because this was an exploratory pilot study designed to inform future studies, all variables were included in the multiple linear regression model to predict quality of life. Follow up analyses using each of the mindfulness subscale scores in a series of multiple linear regressions were conducted to examine whether each subscale score, along with stage of cancer, psychological distress, cognitive fusion, and resilience predicted quality of life.

\section{Results}

Descriptive statistics of the measures are presented in Table 2. Bivariate correlations are reported in Table 3. List-wise deletion was used to address missing data and thus account for the varying sample sizes across the statistics reported in Tables 2-3.

The overall model explained $79.1 \%$ of the total variance in quality of life, $F(5,14)=15.400, p<$ .001 , Adjusted $\mathrm{R}^{2}=.791$. In support of the hypotheses, higher resilience significantly predicted higher quality of life $(b=2.392, p=$ $.002)$, whereas higher stage of cancer $(b=-8.068$, $p=.030)$ and higher levels of psychological distress $(\mathrm{b}=-1.737, p<.001)$ significantly predicted lower quality of life. Contrary to the hypotheses, higher levels of mindfulness significantly predicted lower quality of life $(b=$ $0.612, p=.022)$. Cognitive fusion did not significantly predict quality of life $(p>.05)$.

Table 3.

Bivariate Correlation Matrix of FACT-B with all Other Factors

\begin{tabular}{rrrrrrr}
\hline & & FFMQ & $\begin{array}{r}\text { CD- } \\
\text { RISC }\end{array}$ & $\begin{array}{r}\text { BSI- } \\
18\end{array}$ & $\begin{array}{r}\text { CFQ- } \\
13\end{array}$ & $\begin{array}{r}\text { Stage } \\
\text { of } \\
\text { Cancer }\end{array}$ \\
\hline $\begin{array}{r}\text { FACT- } \\
\text { B }\end{array}$ & r & 0.527 & 0.639 & -0.774 & -0.581 & -0.506 \\
& & & & & & \\
& & 0.012 & 0.001 & 0.000 & 0.005 & 0.016 \\
& $\mathrm{~N}$ & 22 & 22 & 20 & 22 & 22 \\
\hline
\end{tabular}

Follow up analyses using each of the mindfulness subscale scores in a series of multiple linear regressions, did not change the inverse relationship nor yield significant results for the describing subscale $(b=-1.282, p=.087)$, the observing subscale $(b=-1.493, p=.055)$, or the non-reactivity to inner experience subscale $(\mathrm{b}=$ $1.539, p$.191). The acting with awareness subscale significantly predicted lower quality of life $(b=-1.407, p=.025)$. Finally, the nonjudging of inner experience demonstrated a positive relationship that was not significant $(b=$ $.720, p=.358)$.

\section{Discussion}


In support of the hypotheses, the findings of the present study indicated that higher resilience significantly predicted higher quality of life among participants, while higher stage of cancer and psychological distress significantly predicted lower quality of life. Contrary to the hypotheses, higher mindfulness significantly predicted lower quality of life and cognitive fusion did not have a significant effect on quality of life.

The finding that higher resilience predicted higher quality of life among participants supports those of previous researchers (Ha et al., 2014; Markovitz et al., 2015; Wu et al., 2015). Because resilience allows individuals to protect their mental health when faced with adversity, it is possible that participants who had higher levels of resilience were able to maintain a higher quality of life despite the life-changing impact of a breast cancer diagnosis (Ristevska-Dimitrovska et al., 2015). Additionally, higher levels of resilience have been shown to be related to better emotional adjustment in women with breast cancer (Markovitz et al., 2015). Thus, women with higher resilience may have been better able to emotionally adjust to their diagnosis, which improved their quality of life.

The finding that higher stage of cancer predicted lower quality of life supports previous findings (Montazeri 2008). Higher stages of cancer have also been linked to higher levels of distress and anxiety among cancer patients and those with higher stages of cancer often require more invasive treatments, which can cause worry and distress, thereby affecting their quality of life (Ganz et al., 2003; Lemieux et al., 2008). Thus, both the psychological and physical repercussions associated with higher stages of breast cancer may collectively contribute to individuals' lowered quality of life.

The findings from the present study also support those of previous researchers who found that patients with high levels of psychological distress also reported lower quality of life (Pyszel, Malyszczak, Pyszel, Andrzejak, \& Szuba, 2006). Feelings of psychological distress are common among breast cancer patients (Hegel et al., 2006) and may interfere with their coping ability and treatment outcomes, lowering their quality of life (Hoffman et al., 2004; Costanzo et al., 2007). Thus, higher levels of psychological distress may interfere with several aspects of breast cancer patients' lives for extended periods of time, ultimately causing them to experience a lower quality of life.

The results of the present study indicated that higher levels of mindfulness predicted lower quality of life. This finding differs from those of previous researchers who have found that increased mindfulness was associated with improved mental health and that the use of mindfulness-based stress reduction meditation led to enhanced quality of life among patients (Grossman et al., 2004; Coffey et al., 2010; Carlson et al., 2003; Lengacher et al., 2009; Witek-Janusek, 2008; Hoffman et al., 2012; Henderson et al., 2012). A possible explanation for this discrepancy is that all measures used in the present study were taken at baseline and participants had not received any mindfulnessbased education or training.

Mindfulness is a psychological process in which sustained attention is focused on one's momentto-moment cognitive processes, without thinking about, comparing, and/or evaluating unrelated mental phenomena that may occur during periods of practice (Grossman et al., 2004). This process may initially feel unnatural and be difficult for individuals to engage in, especially without training. In addition, maintaining mindful awareness of one's moment-to-moment experience can be uncomfortable when the individual is experiencing considerable emotional and/or physical discomfort. Moreover, the follow-up analyses indicated that acting with awareness was a component of mindfulness that notably predicted lower quality of life. 
Participants of the current study had recently been diagnosed with breast cancer and undergone treatment; for some, this may have been an especially traumatic and difficult time. Acting with awareness and sustained attention to the emotions of the present moment may bring up unwanted and challenging meta-emotions for patients, which may negatively impact their overall experience. The concept of metaemotions (i.e., emotional reaction to one's own emotions) has been examined and found to contribute more notably to one's well-being above and beyond mindfulness (Mitmansgruber, Beck, Hofer, \& Schubler, 2009). It has been proposed that reducing negative meta-emotions (e.g., anxiety about one's emotions) and learning to adopt an accepting stance to one's presentmoment emotions may contribute improved outcomes. Moreover, studies show that learning to respond to one's self in a compassionate way has been shown to positively impact well-being (Neff, 2003; Gilbert, Baldwin, Irons, Baccus, \& Palmer, 2006).

The results of the current study indicated that cognitive fusion did not significantly predict quality of life among participants. This finding differs from those of previous researchers who found that increased cognitive fusion was linked to lower quality of life (Gillanders et al., 2015; Hayes et al., 2006; Fergus \& Bardeen, 2016). Increased cognitive fusion may cause individuals to be highly attached to their thoughts, and their thoughts to be closely connected with their experiences (Solé et al., 2016). The current measure (CFQ-13) is limited in the ability to capture the specific thoughts that patients may be fused with and there is no currently-available measure to assess cognitive fusion with cancerspecific thoughts. Future research is needed to identify whether cognitive fusion with specific categories of thoughts (e.g., catastrophizing, worry thoughts, self-critical thoughts, hopeless thoughts) might differentially relate to quality of life and present-moment coping. Of note, it has been shown that cognitive fusion is related to psychological inflexibility and distress (Hayes et al., 2006; Fergus \& Bardeen, 2016). This may have made the construct statistically redundant with psychological distress, which was also measured, causing cognitive fusion to have no significant effect on participants' quality of life.

As in all studies, there were limitations. One limitation was the small sample size. This was a pilot study and a large sample size was not possible. Additionally, the sample was comprised of women who were mostly White, collegeeducated, and had an annual household income of $\$ 80,000$ or higher; this may limit the generalizability of these findings. Moreover, the eligibility criteria specifying that patients received their diagnoses and treatment within the previous year may have also affected generalizability. Finally, the data were collected via self-report questionnaires and may have been affected by response biases.

\section{Conclusion}

The findings from the present study provided evidence that stage of cancer, psychological distress, mindfulness, and resilience were related to quality of life among breast cancer patients. Additional research with larger and more diverse participants is needed to fully understand the ways in which these factors impact individual's long-term quality of life.

As advancements in medicine and technology improve the treatment outcomes and survival rates of affected individuals, the importance of developing resources and effective therapeutic interventions to serve this population is crucial. By striving to gain a comprehensive understanding of the physical and psychological factors that impact breast cancer patients' overall health and well-being, it may be possible to develop and tailor additional resources to maximize the benefits patients receive from treatment. 


\section{References}

American Cancer Society. (2017). How common is breast cancer? American Cancer Society. Retrieved from https://www.cancer.org/cancer/breast-cancer/about/how-common-is-breast-cancer.html

Baer, R. A., Smith, G. T., Lykins, E., Button, D., Krietemeyer, J., Sauer, S., Walsh, E., Duggan, D., \& Williams, J. M. G. (2008). Construct validity of the five-facet mindfulness questionnaire in meditating and nonmeditating samples. Assessment, 15(3), 329-342. doi: 10.1177/1073191107313003

Brady, M. J., Cella, D. F., Mo, F., Bonomi, A. E., Tulsky, D. S., Lloyd, S. R., Deasy, S., Cobleigh, M. \& Shiomoto, G. (1997). Reliability and validity of the functional assessment of cancer therapybreast quality-of-life instrument. Journal of Clinical Oncology, 15(3), 974-986. doi: 10.1200/JCO.1997.15.3.974.

Campbell-Sills, L. \& Stein, M. B. (2007). Psychometric analysis and refinement of the connor-davidson resilience scale (CD-RISC): Validation of a 10-item measure of resilience. Journal of Traumatic Stress, 20, 1019-1028. doi: 10.1002/jts.20271.

Carlson, L. E., Speca, M., Patel, K. D., \& Goodey, E. (2003). Mindfulness-based stress reduction in relation to quality of life, mood, symptoms of stress, and immune parameters in breast and prostate cancer outpatients. Psychosomatic Medicine, 65(4), 571-581. doi: 10.1097/01.psy.0000074003.35911.41.

Carver, C. S., Smith, R. G., Petronis, V. M., \& Antoni, M. H. (2006). Quality of life among long- term survivors of breast cancer: Different types of antecedents predict different classes of outcomes. Psycho-Oncology: Journal of the Psychological, Social and Behavioral Dimensions of Cancer, 15(9), 749-758. doi: 10.1002/pon.1006

Coffey, K. A., Hartman, M., \& Fredrickson, B. L. (2010). Deconstructing mindfulness and constructing mental health: Understanding mindfulness and its mechanisms of action. Mindfulness, 1(4), 235253. doi: 10.1007/S12671-010-0033-2

Costanzo, E. S., Lutgendorf, S. K., Mattes, M. L., Trehan, S., Robinson, C. B., Tewfik, F., \& Roman, S. L. (2007). Adjusting to life after treatment: Distress and quality of life following treatment for breast cancer. British Journal of Cancer, 97(12), 1625. doi: 10.1038/sj.bjc.6604091.

Derogatis, L. R. (2000). Brief symptom inventory 18. Minneapolis, MN: NCS Pearson. website, https://www.worldcat.org/title/bsi-18-brief-symptom-inventory-18-administration-scoring-andprocedures-manual/oclc/132717179

Fergus, T. A. \& Bardeen, J. R. (2016). Negative mood regulation expectancies moderate the association between happiness emotion goals and depressive symptoms. Personality and Individual Differences, 100, 23-27. doi: 10.1177/01461672962210005

Ganz, P. A., Desmond, K. A., Leedham, B., Rowland, J. H., Meyerowitz, B. E., \& Belin, T. R. (2002). Quality of life in long-term, disease-free survivors of breast cancer: A follow-up study. Journal of the National Cancer Institute, 94(1), 39-49. doi: 10.1093/jnci/94.1.39.

Ganz, P. A., Greendale, G. A., Petersen, L., Kahn, B., \& Bower, J. E. (2003). Breast cancer in younger women: Reproductive and late health effects of treatment. Journal of Clinical Oncology, 21(22), 4184-4193. doi: 10.1200/JCO.2003.04.196. 
Gilbert, P., Baldwin, M. W., Irons, C., Baccus, J. R., \& Palmer, M. (2006). Self-criticism and selfwarmth: An imagery study exploring their relation to depression. Journal of Cognitive Psychotherapy, 20, 183-200. doi: 10.1891/jcop.20.2.183

Gillanders, D. T., Bolderston, H., Bond, F. W., Dempster, M., Flaxman, P. E., Campbell, L., Kerr, S., Tansey, L., Noel, P., Ferenbach, C., Masley, S., Roach, L., Lloyd, J., May, L., Clarke, S., Remington, B. (2014). The development and initial validation of the cognitive fusion questionnaire. Behavior Therapy, 45(1), 83-101. doi: 10.1016/j.beth.2013.09.001.

Gillanders, D. T., Sinclair, A. K., MacLean, M., \& Jardine, K. (2015). Illness cognitions, cognitive fusion, avoidance and self-compassion as predictors of distress and quality of life in a heterogenous sample of adults, after cancer. Journal of Contextual Behavioral Science, 4(4), 300-311. doi: https://doi.org/10.1016/j.jcbs.2015.07.003

Grossman, P., Niemann, L., Schmidt, S., \& Walach, H. (2004). Mindfulness-based stress reduction and health benefits: A meta-analysis. Journal of Psychosomatic Research, 57(1), 35-43. doi: 10.1016/S0022-3999(03)00573-7.

Ha, B. Y., Jung, E. J., \& Choi, S. Y. (2014). Effects of resilience, post-traumatic stress disorder on the quality of life in patients with breast cancer. Korean Journal of Women Health Nursing, 20(1), 83-91. doi: 10.4069/kjwhn.2014.20.1.83.

Härtl, K., Engel, J., Herschbach, P., Reinecker, H., Sommer, H., \& Friese, K. (2010). Personality traits and psychosocial stress: Quality of life over 2 years following breast cancer diagno- sis and psychological impact factors. Psycho-Oncology: Journal of the Psychological, Social and Behavioral Dimensions of Cancer, 19(2), 160-169. doi: 10.1002/pon.1536.

Hayes, S. C., Luoma, J., Bond, F., Masuda, A., \& Lillis, J. (2006). Acceptance and commitment therapy: Model, processes, and outcomes. Behaviour Research and Therapy, 44(1), 1-25. doi: 10.1016/j.brat.2005.06.006.

Hegel, M. T., Moore, C. P., Collins, E. D., Kearing, S., Gillock, K. L., Riggs, R. L., Clay, K. T., \& Ahles, T. A. (2006). Distress, psychiatric syndromes, and impairment of function in women with newly diagnosed breast cancer. Cancer, 107(12), 2924-2931. doi: 10.1002/cncr.22335.

Henderson, V. P., Clemow, L., Massion, A. O., Hurley, T. G., Druker, S., \& Hébert, J. R. (2012). The effects of mindfulness-based stress reduction on psychosocial outcomes and quality of life in early-stage breast cancer patients: a randomized trial. Breast Cancer Research and Treatment, 131(1), 99-109. doi: 10.1007/s10549-011-1738-1.

Hoffman, C. J., Ersser, S. J., Hopkinson, J. B., Nicholls, P. G., Harrington, J. E., \& Thomas, P. W. (2012). Effectiveness of mindfulness-based stress reduction in mood, breast-and endocrine-related quality of life, and well-being in stage 0 to III breast cancer: a randomized, controlled trial. Journal of Clinical Oncology, 30(12), 1335-1342. doi: 10.1200/JCO.2010.34.0331.

Hoffman, B. M., Zevon, M. A., D’Arrigo, M. C., \& Cecchini, T. B. (2004). Screening for distress in cancer patients: The NCCN rapid-screening measure. Psychooncology, 13(11), 792-9. doi: 10.1002/pon.796.

Kershaw, T., Northouse, L., Kritpracha, C., Schafenacker, A., \& Mood, D. (2004). Coping strategies and quality of life in women with advanced breast cancer and their family caregivers. Psychology \& Health, 19(2), 139-155. doi: 10.1080/08870440310001652687

Kröger, M. J., Menzel, T., Gschwend, J. E., \& Bergmann, L. (1999). Life quality of patients with metastatic renal cell carcinoma and chemo-immunotherapy--a pilot study. Anticancer research, 19(2C), 1553-1555. Website, https://pubmed.ncbi.nlm.nih.gov/10365144/ 
Lemieux, J., Maunsell, E., \& Provencher, L. (2008). Chemotherapy-induced alopecia and effects on quality of life among women with breast cancer: a literature review. Psycho-Oncology, 17(4), 317-328. doi: 10.1002/pon.1245.

Lengacher, C. A., Johnson-Mallard, V., Post-White, J., Moscoso, M. S., Jacobsen, P. B., Klein, T. W., Widen, R. H., Fitzgerld, S. G., Shelton, M. M., Barta, M., Goodman, M., Cox, C. E., \& Kip, K. E. (2009). Randomized controlled trial of mindfulness-based stress reduction (MBSR) for survivors of breast cancer. Psycho-Oncology, 18(12), 1261-1272. doi: 10.1002/pon.1529.

Markovitz, S. E., Schrooten, W., Arntz, A., \& Peters, M., L. (2015). Resilience as a predictor for emotional response to the diagnosis and surgery in breast cancer patients. Psycho- Oncology, 24(12), 1639-1645. doi: 10.1002/pon.3834.

McCracken, L. M., DaSilva, P., Skillicorn, B. D., \& Doherty, R. (2014) The cognitive fusion questionnaire: A preliminary study of psychometric properties and prediction of functioning in chronic pain. The Clinical Journal of Pain, 30(10), 894-901. doi: 10.1097/AJP.0000000000000047.

Mitmansgruber, H., Beck, T. N., Höfer, S., \& Schüßler, G. (2009). When you don’t like what you feel: Experiential avoidance, mindfulness and meta-emotion in emotion regulation. Personality and Individual Differences, 46(4), 448-453. doi: 10.1016/j.paid.2008.11.013

Mols, F., Vingerhoets, A. D., Coebergh, J. W., \& Van de Poll-Franse, L V. (2005). Quality of life among long-term breast cancer survivors: A systematic review. European Journal of Cancer, 41(17), 2613-2619. doi: 10.1016/j.ejca.2005.05.017.

Montazeri, A. (2008). Health-related quality of life in breast cancer patients: A bibliographic review of the literature from 1974 to 2007. Journal of Experimental \& Clinical Cancer Research, 27(1), 32. doi: 10.1186/1756-9966-27-32.

National Breast Cancer Foundation, Inc. (2016). Breast cancer stages. National Breast Cancer Foundation, Inc. Retrieved from http://www.nationalbreastcancer.org/breast-cancer-stages

Neff, K. D. (2003). The development and validation of a scale to measure self compassion. Self and Identity, 2, 223-250. doi: 10.1080/15298860390209035

Pyszel, A., Malyszczak, K., Pyszel, K., Andrzejak, R., \& Szuba, A. (2006). Disability, psychological distress and quality of life in breast cancer survivors with arm lymphedema. Lymphology, 39(4), 185-192. website, https://pubmed.ncbi.nlm.nih.gov/17319631/

Ristevska-Dimitrovska, G., Filov, I., Rajchanovska, D., Stefanovski, P., \& Dejanova, B. (2015). Resilience and quality of life in breast cancer patients. Open Access Macedonian Journal of Medical Sciences, 3(4), 727-31. doi: 10.3889/oamjms.2015.128.

Solé, E., Racine, M., Castarlenas, E., de la Vega, R., Tomé-Pires, C., Jensen, M., \& Miró, J. (2016). The psychometric properties of the cognitive fusion questionnaire in adolescents. European Journal of Psychological Assessment, 32(3), 181-186. doi: 10.1027/1015-5759/a000244

Stanton, A., Danoff-Burg, S., Cameron, C., Bishop, M., Collins, C., Kirk, S., Sworowski, L., \& Twillman, R. (2000). Emotionally expressive coping predicts psychological and physical adjustment to breast cancer. Journal of Consulting and Clinical Psychology, 68(5), 875-882. doi: 10.1037//0022-006x.68.5.875

U.S. Breast Cancer Statistics. (2018, January 09). Retrieved April 02, 2018, from http:// www.breastcancer.org/symptoms/understand bc/statistics.

Witek-Janusek, L., Albuquerque, K., Chroniak, K. R., Chroniak, C., Durazo-Arvizu, R., \& Mathews, H. L. (2008). Effect of mindfulness based stress reduction on immune function, quality of life and 
coping in women newly diagnosed with early stage breast cancer. Brain, Behavior, and Immunity, 22(6), 969-981. doi: 10.1016/j.bbi.2008.01.012.

Wu, W. W., Tsai, S. Y., Liang, S. Y., Liu, C. Y., Jou, S. T., \& Berry, D. L. (2015). The mediating role of resilience on quality of life and cancer symptom distress in adolescent patients with cancer. Journal of Pediatric Oncology Nursing, 32(5), 304-313. doi: $10.1177 / 1043454214563758$.

Xie, Y., Peng, L., Zuo, X., \& Li, M. (2016). The psychometric evaluation of the Connor-Davidson Resilience Scale using a Chinese military sample. PLoS ONE, 11(2), e0148843. http://doi.org/10.1371/journal.pone.0148843

Zabora, J., Brintzenhofeszoc, K., Curbow, B., Hooker, C., \& Piantadosi, S. (2001). The prevalence of psychological distress by cancer site. Psycho-Oncology, 10(1), 19-28. doi: 10.1002/10991611(200101/02)10:1<19::aid-pon501>3.0.co;2-6.

Zebrack, B. J., Yi, J., Petersen, L., \& Ganz, P. A. (2008). The impact of cancer and quality of life for long-term survivors. Psycho-Oncology, 17(9), 891-900. doi: 10.1002/pon.1300.

Corresponding Author Information

Terry A. Cronan

tcronan@sdsu.edu

phone: (619) 594-6915

Fax: (619) 594-1247 